\title{
Establishing a Canadian global surgery community: a national survey
}

\section{Kim, D; Wong, H; Fawcett, V; Hameed, M; Westerberg, B; Ball-Banting, E; Joos, E University of British Columbia}

\section{Introduction}

- Surgical care is an indispensable but often neglected part of global health

- Worldwide, an estimated 5 billion people lack access to safe surgery and/or anesthesia

- Many organizations for global surgical care exist, but coordination of organization initiatives for efficient, sustainable response is often lacking

\section{Methods}

- Global Surgery Offices (GSOs) in Canada were identified through word of mouth and CAGS

- Electronic surveys and phone interviews were performed to determine:

Resources available

Research areas of involvement

Countries of interest

\section{Results}

- Seven academic institutions have GSOs

- Six responded:

UBC, Alberta, Calgary, McMaster, McGill, Dalhousie

Number of countries that each academic institution's GSO is involved in, sorted by continent



Number of GSOs involved in each field of work

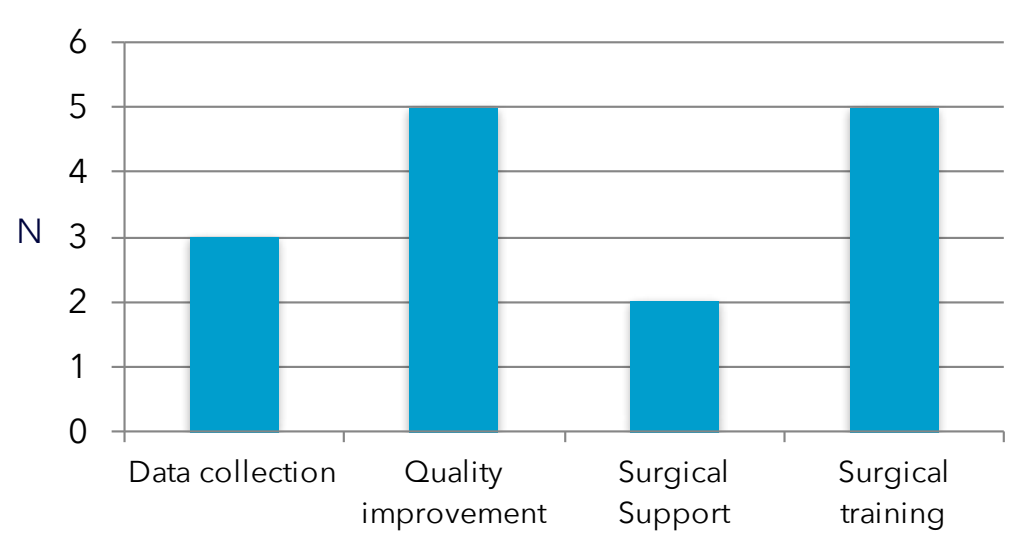

Number of GSOs receiving funding from each source

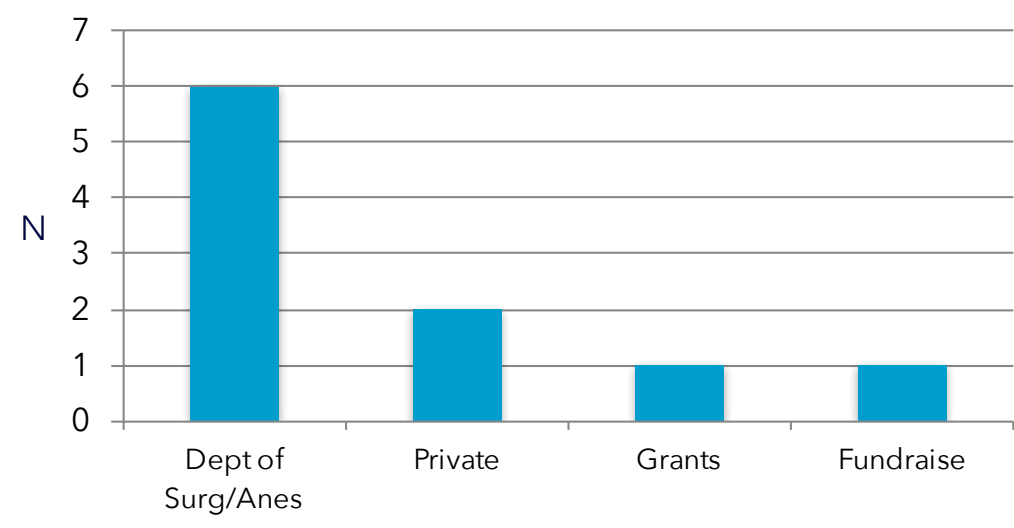

\section{Conclusion}

- Canadian GSOs work independently but in similar areas

- This survey provides an initial framework for a unified "Canadian Global Surgery Initiative Group (CGSIG)"

- Such a future initiative would benefit from inclusion of other groups, i.e. NGOs, private organizations 